\title{
Divergência genética em trigo de sequeiro por meio de caracteres morfoagronômicos
}

\author{
Aurinelza Batista Teixeira Condé ${ }^{1}$, Maurício Antônio de Oliveira Coelho ${ }^{1}$, Vanoli Fronza ${ }^{2}$, \\ Leandro Vagno de Souza ${ }^{3}$
}

\begin{abstract}
RESUMO
O cultivo de trigo de sequeiro constitui ótima opção para o Estado de Minas Gerais em regiões com altitude superior a $800 \mathrm{~m}$. Neste estudo objetivou-se estimar a divergência genética entre genótipos de trigo com o auxílio de métodos de análise multivariada, objetivando identificar potenciais genótipos para obtenção de combinações híbridas promissoras. Foi utilizado o delineamento experimental em blocos ao acaso, com quatro repetições. Avaliaram-se seis caracteres morfoagronômicos em 11 genótipos de trigo cultivados em condições de sequeiro no município de Patos de Minas-MG, nos anos de 2007 e 2008. Houve grande variabilidade fenotípica entre os genótipos de trigo avaliados em cultivo de sequeiro. Os genótipos IAC 289-L22 e IAC 289-L4 foram os mais similares, enquanto os CD 113 e EP 011106, os maisdivergentes, promissores nos cruzamentos para obtenção de materiais adaptados e com bons atributos fenotípicos emcultivo de sequeiro.
\end{abstract}

Palavras-chave: Triticum aestivum L., análise multivariada, dissimilaridade.

\begin{abstract}
Genetic divergence in rainfed wheat based on morphoagronomic traits

Wheat cultivation in rainfed conditions is a good option for regions in Minas Gerais with altitudes above $800 \mathrm{~m}$. The objective of this study was to estimate the genetic diversity of eleven wheat genotypes grown in rainfed conditions, using multivariate analysis based on six morpho-agronomic traits to identify which genotypes have potential for hybrid combinations. The treatments were arranged in a randomized complete block design with four replicates. The experiments were carried out during the wheat cropping season 2007/2008. Great variability was found among the wheat genotypes evaluated in rainfed conditions. The genotypes IAC 289-L22 and IAC 289-L4 were the most similar. The genotypes CD 113 and EP 011106 were the most diverging and the most promising for breeding towards generating material adapted and with good characteristics for rainfed cultivation.
\end{abstract}

Key words: Triticum aestivum L., dissimilarity , multivariate analysis.

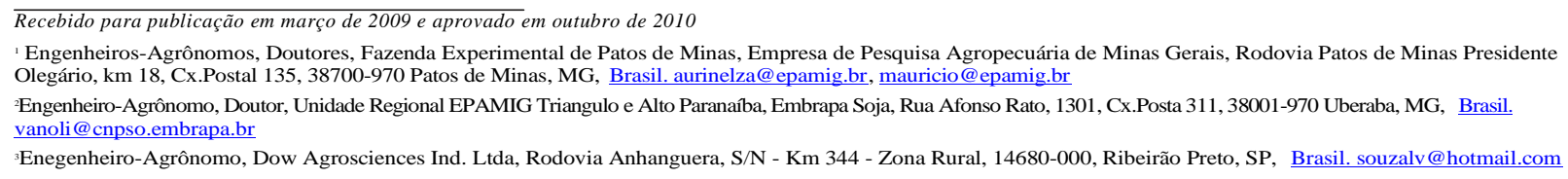




\section{INTRODUÇÃO}

A região do cerrado de Minas Gerais constitui ótima alternativa para a expansão da produção tritícola, pois existe a possibilidade de colheita em períodos de quase ausência de pluviosidade, o que proporciona a obtenção de um produto de alta qualidade, cujo peso hectolítrico médio, normalmente, é maior que $80 \mathrm{~kg} / \mathrm{hL}$, superior ao padrão de $78 \mathrm{~kg} / \mathrm{hL}$ exigido pela legislação (Brasil, 2001). A baixa umidade relativa do ar durante a maior parte do ciclo da culturatambém contribui para a diminuição da ocorrência de doenças, o que torna o trigo um atrativo aos agricultores daregião central do Brasil.

O cultivo do trigo de sequeiro é uma opção no Estado de Minas Gerais em áreas com altitude superior a $800 \mathrm{~m}$, e tem apresentado outras características desejáveis, como alto potencial produtivo, diminuição da população de plantas daninhas para a próxima cultura de verão, fornecimento de cobertura morta de longa duração (elevada relação $\mathrm{C} / \mathrm{N}$ ) para as áreas de plantio direto e proximidade dos moinhos (Fronza et al., 1999).

Variabilidade genética é uma condição imprescindível para a formação de populações segregantes em programas de melhoramento genético e pode ser útil at6 na recomendação de cultivares para determinadas regiões quando o objetivo é aumentar a base genética dos cultivares a serem indicados aos agricultores (Bertan et al., 2007). Além disso, a quantificação da dissimilaridade genética pode servir como parâmetro para identificação de genitores que possibilitem maior efeito heterótico na progênie e maior possibilidade de recuperação de recombinantes superiores nas gerações segregantes (Bertan et al., 2006).

As técnicas de análise multivariada têm sido utilizadas rotineiramente no estudo da divergência genética, pois consideram, simultaneamente, as variáveis avaliadas dos genótipos, além da correlação existente entre elas. Para obter as medidas de dissimilaridades, vários autores (Teixeira et al., 2004; Chiorato et al., 2005; Bertan et al., 2006) têm utilizado a distância generalizada de Mahalanobis. Com essa medida é possível proceder à analise de agrupamento dos genótipos, pelo método de otimização de Tocher, que proporciona resultados muito conclusivos sobre a diversidade e permite a indicação de cruzamentos promissores para futuros programas de melhoramento envolvendo os genótipos avaliados.

Nesse contexto, o objetivo deste estudo foi estimar a divergência genética entre 11 genótipos de trigo cultivados em condições de sequeiro, com base em seis variáveis morfoagronômicas, em duas safras agrícolas, com o intuito de identificar genótipos que tenham potencial para combinações híbridas promissoras.

\section{MATERIAL E MÉTODOS}

Os experimentos foram conduzidos na Fazenda Experimental de Sertãozinho (FEST), pertencente à Empresa de Pesquisa Agropecuária de Minas Gerais (EPAMIG), loca lizada em Patos de Minas-MG, a 940 m de altitude, latitude de $18^{\circ} 36^{\prime}$ 'S e longitude de $46^{\circ} 31^{\prime} \mathrm{W}$, de 9 de março a 25 de junho de 2007 e de 17 de março a $1^{\circ}$. de julho de 2008 , em condições de sequeiro. Os experimentos foram constituídos de seis cultivares indicados para Minas Gerais e de cinco linhagens em fase final de avaliação, em ensaio de rendimento para o cultivo de sequeiro. Os genótipos utilizados estão apresentados na Tabela 1.

O delineamento experimental utilizado foi o de blocos ao acaso, com quatro repetições. Cada parcela apresentou 5,0 m de comprimento e 1,0 m de largura, sendo constituídas por cinco linhas, espaçadas de $0,20 \mathrm{~m}$ e com cerca de 400 sementes viáveis $/ \mathrm{m}^{2}$ na semeadura. Na colheita, consideraram-se as três linhas centrais como área útil $\left(3,0 \mathrm{~m}^{2}\right)$.

Foram avaliadas as seguintes variáveis morfoagronômicas: 1) altura da planta, que foi obtida no momento da colheita, considerando-se a distância, em centímetros, do nível do solo ao ápice da espiga, com exclusão das aristas, e estimando a média de diferentes pontos de cada parcela; 2) rendimento de grãos, em gramas, obtido pesando-se a produção total de cada parcela útil, a qual foi transformada para $\mathrm{kg} / \mathrm{ha}$; 3 ) número de dias decorridos da emergência das plantas ao espigamento, considerando-se pelo menos $50 \%$ das plantas com as espigas totalmente para fora da bainha da folha bandeira; 4) porcentagem de plantas acamadas, mediante avaliação visual no momento da colheita, em escala que variou de 0 (sem acamamento) at6 $100 \%$ (completamente acamadas); 5) valor agronômico da parcela, mensurado por meio de escala de notas que variou de 1 (péssimo) a 5 (ótimo), considerando-se o aspecto geral das plantas no momento da colheita; e 6) peso hectolitrico, medido a partir de uma amostra aleatória dos grãos de cada parcela, realizado conforme procedimento descrito na Instrução Normativa SARC No 7, de 15/08/2001, do Minist6rio da Agricultura e do Abastecimento (Brasil, 2001), utilizando balança marca Dalle Molle.

Após a coleta dos dados foram empregadas analises genético estatísticas, utilizando-se o programa Genes (Cruz, 2001). Inicialmente foram realizadas as analises de variância individuais para cada safra agrícola, e posteriormente, as analises de variância conjunta, considerando o modelo misto com efeito de ambiente fixo (Cruz \& Carneiro, 2003).

A comparação das médias foi realizada utilizando-se o teste de agrupamento proposto por Scott-Knott (1974) a $5 \%$ de significância. A distância genética entre todos os pares de genótipos foi estimada por meio da distância generalizada de Mahalanobis e pelo método de análise de variáveis canônicas. Com base na matriz de dissimilaridade genética gerada foi aplicado o método de agrupamento de Tocher (Rao, 1952).

\section{RESULTADOS E DISCUSSÃO}

$\mathrm{Na}$ Tabela 2 estão apresentadas as precipitações totais e as médias das temperaturas máximas e mínimas dos meses correspondentes aos dois períodos em que foram 
conduzidos os experimentos. De maneira geral, no ano de 2008 as condições climáticas foram mais favoráveis para a expressão do potencial produtivo dos genótipos do que no ano de 2007, principalmente em termos de disponibilidade hídrica.

Nas análises de variância foram observadas diferenças significativas entre os genótipos para todas as variáveis avaliadas em ambos os anos de estudo, o que é uma condição importante para o estudo da divergência genética entre os genótipos. Isso também demonstra a possibilidade de selecionar genótipos superiores para serem indicados na região do cerrado mineiro para cultivo em condições de sequeiro.

Todas as variáveis avaliadas apresentaram diferenças significativas, com exceção da produção de grãos para o efeito da interação genótipo x ambiente. Este resultado demonstra que ha respostas diferenciadas dos genótipos com a mudança do ambiente, que pode ser consequência, por exemplo, do clima e do índice de precipitação pluviométrica entre os dois anos de cultivo. No ano de 2008, com maiores índices pluviométricos, foi possível obter maiores valores médios para todas as características avaliadas (Tabela 3).

Os coeficientes de variação dos caracteres avaliados em 2007 oscilaram entre 0,79\% (peso hectolítrico) e 16,78\% (produção de grãos), indicando boa precisão experimental, embora para produção de grãos o valor tenha sido próximo a 20\%, que 6 o limite Maximo estabelecido pelo Minist6rio da Agricultura, Pecuária e Abastecimento (MAPA) para ensaios de rendimento visando avaliar o valor de cultivo e o uso de cultivares no Brasil (Brasil, 2001). Porém, por se tratar de ensaio de trigo de sequeiro e o ano agrícola de 2007 ter apresentado ocorrência de severa restrição hídrica às plantas (Tabela 2 ), esse valor esta dentro da faixa esperada. No ano agrícola de 2008 os coeficientes de variação dos caracteres estiveram entre $0,99 \%$ (peso hectolítrico) e $15,12 \%$ (valor agronômico). Em ambos os anos, embora tenha sido avaliada a porcentagem de acamamento, não foi realizada a analise de variância, pois, pela analise de resíduos feita previamente, os dados não atenderam às pressuposições da analise de variância e ao coeficiente de variação, mesmo com o uso da transformação dos dados.

Segundo Cavariani \& Souza (1983), os cultivares precoces atingem o espigamento, em média, com 50 dias (genótipos que formaram o grupo “c” em 2007 e "f” em

Tabela 1. Genótipos de trigo utilizados, cruzamento, origem, instituição responsável e região onde é indicada para cultivo

\begin{tabular}{|c|c|c|c|c|}
\hline Genótipos & Cruzamento & Origem & Instituição & Região de indicação \\
\hline CD 105 & $\begin{array}{c}\text { PFAU “S”/2*OCEPAR } \\
\text { 14//IAPAR } 41\end{array}$ & Brasil & Coodetec & $\begin{array}{l}\text { RS, SC, PR, MS, SP, } \\
\text { MG, GO, DF e MT }\end{array}$ \\
\hline CD 111 & $\begin{array}{l}\text { EMBRAPA 27/OCEPAR } \\
\text { 18//ANAHUAC } 75\end{array}$ & Brasil & Coodetec & $\begin{array}{l}\text { RS, SC, PR, MS, SP, } \\
\text { MG, GO, DF e MT }\end{array}$ \\
\hline CD 113 & IOIIAIVATUAC II & Brasil & Coodetec & RS, SC, PR, MS, SP, \\
\hline EP 011106 & EMBRAPA 27/OC 946 & M6xico & EPAMIG & MG, GO, DF e MT \\
\hline EP 011187 & PASTOR/3/VEE\#5//DOVE/BUC & M6xico & EPAMIG & linhagem \\
\hline EP 011210 & WEEBILL1 & M6xico & EPAMIG & linhagem \\
\hline IAC 289-L4 & $\begin{array}{c}\text { BABAX*2/PRL } \\
\text { Selegão em IAC 289-MARRUÁ }\end{array}$ & Brasil & COOPADAP $^{1}$ & linhagem \\
\hline IAC 289-L22 & (KVZ/BUHO//KAL/BB) & Brasil & COOPADAP $^{1}$ & linhagem \\
\hline IAC 350-Goiapa & $\begin{array}{c}\text { Selegão em IAC 289-MARRUÁ } \\
(\mathrm{KVZ/BUHO//KAL/BB)}\end{array}$ & M6xico & IAC & linhagem \\
\hline MGS1 Alianga & 2109-36/SERI & Brasil & EPAMIG & SP e MG \\
\hline MGS Brilhante & $\begin{array}{l}\text { PF 858/OCEPAR } 11 \\
\text { PF 8640/BR } 24\end{array}$ & Brasil & EPAMIG & $\begin{array}{l}\text { DF, GO, MG e MT } \\
\text { DF, GO e MG }\end{array}$ \\
\hline
\end{tabular}

Cooperativa Agropecuária do Alto Paranaíba, São Gotardo-MG

Tabela 2 . Precipitação total e médias de temperatura (máxima e mínima) mensais no período de condução dos ensaios nos anos de 2007 e $2008^{1}$

\begin{tabular}{lcccccc}
\hline \multirow{2}{*}{ Mês } & \multicolumn{3}{c}{ Ano de 2007 } & \multicolumn{2}{c}{ Ano de 2008 } \\
\cline { 2 - 7 } & Prec. $(\mathbf{m m})$ & Máx. $\left({ }^{\circ} \mathbf{C}\right)$ & Mín. $\left({ }^{\circ} \mathbf{C}\right)$ & Prec. $(\mathbf{m m})$ & Máx. $\left({ }^{\circ} \mathbf{C}\right)$ & Mín. $\left({ }^{\circ} \mathbf{C}\right)$ \\
\hline Margo & 38,2 & 29,4 & 16,4 & 105,4 & 28,6 & 17,8 \\
Abril & 46,6 & 30,7 & 16,2 & 112,7 & 29,0 & 17,0 \\
Maio & 2,4 & 28,4 & 14,2 & 23,8 & 26,6 & 13,5 \\
Junho & 0,8 & 27,8 & 11,8 & 32,6 & 26,6 & 13,6 \\
\hline
\end{tabular}

INMET - Estação Meteorológica de Patos de Minas, MG. 
2008) (Tabela 3), os de ciclo médio com 56 dias (genótipos que formaram os grupos "a" e "b" em 2007 e "d" e "e" em 2008) e os de ciclo tardio com 62 dias (genótipos que formaram os grupos "a", "b" e "c" em 2008). Nos dois anos de avaliação, o cultivar CD 113 e a linhagem EP 011106 foram os genótipos mais precoces, e os cultivares MGS Brilhante e CD 111, os mais tardios. Porém, nenhum genótipo apresentou ciclo demasiadamente longo para o cultivo em sequeiro nos cerrados.

A altura das plantas de trigo é uma característica importante, considerando sua associação com o potencial de rendimento de grãos e a resistência ao acamamento (Lobato, 2006), além de proporcionar melhor colheita mecanizada, utilização de maior densidade de plantas e maior quantidade de adubo (Bered et al., 2002). Os genótipos avaliados apresentaram estatura muito baixa no ano de 2007, inferior a $72 \mathrm{~cm}$ (Tabela 3). No ano de 2008, as plantas tiveram estatura que variou de 60 a $84 \mathrm{~cm}$ e, embora maiores, ainda são consideradas de porte baixo, podendo ser classifica das como porte semianão. Da mesma forma, em 2008 a ocor- rência de acamamento foi maior que em 2007, principalmente no cultivar IAC 350 e nas linhagens IAC 289-L22 e IAC 289-L4. Merece destaque o cultivar MGS Brilhante, o qual, apesar de ser o mais alto em ambos os anos, apresentou elevada resistência ao acamamento.

Quanto à característica valor agronômico, a maioria dos genótipos, no ano de 2007, recebeu notas iguais ou maiores que 3,0, exceto as linhagens EP 011187 e EP 011210, que ficaram em grupos à parte e que também apresentaram baixa produtividade. Essas linhagens, no ano de 2008, com melhores condições pluviométricas, ficaram no grupo dos genótipos com maior valor agronômico, demonstrando como o seu comportamento foi altamente influenciado pelas variações das condições ambientais. Os demais genótipos apresentaram produtividade média próxima à média nacional, que é de $2.000 \mathrm{~kg} / \mathrm{ha}(\mathrm{CONAB}$, 2008). No ano de 2008, os genótipos também receberam notas maiores que 3,0 , na sua maioria, exceto os cultiva-

Tabela 3. Médias ${ }^{(1)}$ dos genótipos de trigo em relação às variáveis ciclo, da emergência ao espigamento (ESP), altura de plantas (ALT), valor agronômico (VA), produção de grãos (PG), peso hectolítrico (PH) e acamamento avaliados nos anos de 2007 e 2008

\begin{tabular}{|c|c|c|c|c|c|c|}
\hline \multicolumn{7}{|c|}{2007} \\
\hline Ganótinne & $\begin{array}{r}\text { ESP } \\
\text { (dias) } \\
\end{array}$ & $\begin{array}{l}\text { ALT } \\
(\mathbf{c m}) \\
\end{array}$ & $\begin{array}{c}\text { VA } \\
\text { (Notas) }\end{array}$ & $\begin{array}{c}\text { PG } \\
\text { (kg/ha) }\end{array}$ & $\begin{array}{c}\text { PH } \\
(\mathrm{kg} / \mathrm{hl}) \\
\end{array}$ & $\begin{array}{c}\text { ACAM } \\
(\%)\end{array}$ \\
\hline MGS 1 Aliança & $54 \mathrm{~b}$ & $66 \mathrm{~b}$ & $3,00 \mathrm{~b}$ & $1917 \mathrm{a}$ & $78,8 \mathrm{c}$ & 1,75 \\
\hline MGS Brilhante & $59 \mathrm{a}$ & $71 \mathrm{a}$ & $3,00 \mathrm{~b}$ & $1635 \mathrm{a}$ & $79,8 \mathrm{~b}$ & 0,00 \\
\hline CD 105 & $55 \mathrm{~b}$ & $63 \mathrm{~b}$ & $4,00 \mathrm{a}$ & $1526 \mathrm{~b}$ & 81,0 a & 1,75 \\
\hline CD 111 & $58 \mathrm{a}$ & $63 \mathrm{~b}$ & $4,00 \mathrm{a}$ & $1682 \mathrm{a}$ & $78,2 \mathrm{c}$ & 0,50 \\
\hline CD 113 & $51 \mathrm{c}$ & $52 \mathrm{c}$ & $3,25 \mathrm{~b}$ & $1451 \mathrm{~b}$ & $78,8 \mathrm{c}$ & 1,00 \\
\hline EP 011106 & $51 \mathrm{c}$ & $62 \mathrm{~b}$ & $4,50 \mathrm{a}$ & $1917 \mathrm{a}$ & $76,7 \mathrm{~d}$ & 1,00 \\
\hline EP 011187 & $54 \mathrm{~b}$ & $62 \mathrm{~b}$ & $2,50 \mathrm{c}$ & $1172 b$ & $73,4 \mathrm{f}$ & 2,25 \\
\hline EP 011210 & $54 \mathrm{~b}$ & $61 \mathrm{~b}$ & $1,75 \mathrm{~d}$ & $1229 \mathrm{~b}$ & $74,8 \mathrm{e}$ & 2,75 \\
\hline IAC 289-L22 & $54 \mathrm{~b}$ & $66 \mathrm{~b}$ & $3,25 \mathrm{~b}$ & $1776 \mathrm{a}$ & $72,2 \mathrm{f}$ & 2,25 \\
\hline IAC 289-L4 & $52 \mathrm{c}$ & $64 \mathrm{~b}$ & $3,00 \mathrm{~b}$ & $1807 \mathrm{a}$ & $72,8 \mathrm{f}$ & 2,00 \\
\hline IAC 350 & $56 \mathrm{~b}$ & $61 \mathrm{~b}$ & $3,75 \mathrm{a}$ & $1781 \mathrm{a}$ & $78,2 \mathrm{c}$ & 5,00 \\
\hline Média & 54 & 63 & 3,27 & 1627 & 76,8 & 1,84 \\
\hline $\mathrm{CV}(\%)$ & 1,93 & 5,09 & 15,14 & 16,78 & 0,79 & - \\
\hline \multicolumn{7}{|c|}{2008} \\
\hline MGS 1 Aliança & $59 \mathrm{~d}$ & $81 \mathrm{a}$ & $2,75 \mathrm{~b}$ & $2834 \mathrm{a}$ & $80,6 \mathrm{a}$ & 8,75 \\
\hline MGS Brilhante & $63 \mathrm{~b}$ & $84 \mathrm{a}$ & $3,00 \mathrm{~b}$ & $2377 \mathrm{~b}$ & $79,8 \mathrm{a}$ & 2,25 \\
\hline CD 105 & $59 \mathrm{~d}$ & $69 \mathrm{c}$ & $3,00 \mathrm{~b}$ & $2814 \mathrm{a}$ & $78,5 \mathrm{~b}$ & 10,25 \\
\hline CD 111 & $68 \mathrm{a}$ & $71 \mathrm{~b}$ & $3,25 \mathrm{~b}$ & $2393 b$ & $80,0 \mathrm{a}$ & 4,00 \\
\hline CD 113 & $53 \mathrm{f}$ & $60 \mathrm{~d}$ & $2,50 \mathrm{~b}$ & $2350 \mathrm{~b}$ & $78,2 \mathrm{~b}$ & 0,75 \\
\hline EP 011106 & $53 \mathrm{f}$ & $68 \mathrm{c}$ & $3,50 \mathrm{~b}$ & $2932 \mathrm{a}$ & $78,6 \mathrm{~b}$ & 1,75 \\
\hline EP 011187 & $60 \mathrm{c}$ & $74 \mathrm{~b}$ & $4,75 \mathrm{a}$ & $2923 \mathrm{a}$ & $78,9 \mathrm{~b}$ & 12,50 \\
\hline EP 011210 & $59 \mathrm{~d}$ & $76 \mathrm{~b}$ & $4,25 \mathrm{a}$ & $2468 \mathrm{~b}$ & $79,1 \mathrm{~b}$ & 7,50 \\
\hline IAC 289-L22 & $57 \mathrm{e}$ & $77 \mathrm{~b}$ & $4,75 \mathrm{a}$ & $2916 \mathrm{a}$ & $73,6 \mathrm{c}$ & 14,50 \\
\hline IAC 289-L4 & $56 \mathrm{e}$ & $77 \mathrm{~b}$ & $4,50 \mathrm{a}$ & $2882 \mathrm{a}$ & $73,5 \mathrm{c}$ & 13,75 \\
\hline IAC 350 & $59 \mathrm{~d}$ & $77 \mathrm{~b}$ & $3,50 \mathrm{~b}$ & $2881 \mathrm{a}$ & $78,9 \mathrm{~b}$ & 17,00 \\
\hline Média & 59 & 74 & 3,61 & 2706 & 78,1 & 8,45 \\
\hline $\mathrm{CV}(\%)$ & 1,93 & 4,64 & 15,11 & 11,68 & 0,99 & - \\
\hline
\end{tabular}

'Médias seguidas pela mesma letra, em cada coluna e em cada ano agrícola considerado, não diferem entre si, a 5\% de probabilidade, pelo teste de Scott-Knott. 
res MGS 1 Aliança e CD 113, mas que ainda permaneceram no mesmo grupo que outros genótipos com nota igual ou superior a 3,0. Com melhores condições ambientais, maior precipitação, todos os genótipos avaliados tiveram média de produtividade maior que $2.000 \mathrm{~kg} / \mathrm{ha}$, variando entre 2.350 (CD 113) e $2.932 \mathrm{~kg} / \mathrm{ha}$ (EP 011106).

Para o peso hectolítrico foram formados seis grupos, no ano de 2007, pelo teste de Skott-Knott (Tabela 3), e os genótipos MGS 1 Aliança, MGS Brilhante, CD 105, CD 111, CD 113 e IAC 350, presentes nos três primeiros grupos, apresentaram valores maiores que o mínimo de $78 \mathrm{~kg} / \mathrm{hL}$ exigido pela legislação brasileira para serem classificados como tipo I (Brasil, 200 1) e não receber desconto durante a comercialização. Em 2008, apenas as linhagens IAC 289L4 e IAC 289-L22 apresentaram peso hectolítrico menor que $78 \mathrm{~kg} / \mathrm{hL}$.

O método de Tocher (Tabela 4) permitiu a distinção de grupos diferenciados nos dois anos de cultivo. No ano de 2008 os grupos formados pelo método de Tocher apre sentaram algumas discordâncias com o os grupos obti- dos no ano de 2007, mas o cultivar CD 113 mostrou-se um dos mais divergentes e as linhagens IAC 289-L22 e IAC 289-L4 foram as mais similares, considerando os dois anos de cultivo. Como estas duas linhagens foram obtidas por seleção dentro do cultivar IAC 289, esta similaridade já era esperada.

Como as duas primeiras variáveis canônicas explicaram mais de $80 \%$ da variância total de caracteres analisados $(87,3$ e $84 \%$ da variância total acumulada nos anos de 2007 e 2008, respectivamente), é possível explicar de maneira satisfatória a variabilidade manifestada entre os genótipos considerados na avaliação (Figuras 1 e 2). No ano de 2007 houve a formação de cinco grupos: Grupo I IAC 289-L22, IAC 289-L4, EP 011187 e EP 011210; Grupo II- EP 011106; Grupo III - CD 105; Grupo IV - CD 111, IAC 350, MGS 1 Aliança e MGS Brilhante; e Grupo V - CD 113. No ano de 2008 houve a formação de seis grupos: Grupo I - IAC 289-L22 e IAC 289-L4; Grupo II - EP 011187, EP

Tabela 4. Grupos de genótipos de trigo estabelecidos pelo método de Tocher, com base na dissimilaridade expressa pela distância de Mahalanobis, em relação às seis variáveis avaliadas nos anos de 2007 e 2008

\begin{tabular}{|c|c|c|c|}
\hline \multicolumn{4}{|c|}{2007} \\
\hline Grupos & \multicolumn{3}{|c|}{ Genótipos } \\
\hline I & IAC 289-L22 & EP 011187 & EP 011210 \\
\hline II & IAC 350 & & \\
\hline III & MGS1 Aliança MGS Brilhante & CD 105 & \\
\hline IV & CD 113 & & \\
\hline $\mathrm{V}$ & EP 011106 & & \\
\hline \multicolumn{4}{|c|}{2008} \\
\hline $\bar{I}$ & IAC 289-L22 $\quad$ IAC 289-L4 & & \\
\hline II & EP 011210 & EP 011187 & MGS1 Aliança CD 105 MGS Brilhante EP 011106 \\
\hline III & CD 113 & & \\
\hline
\end{tabular}

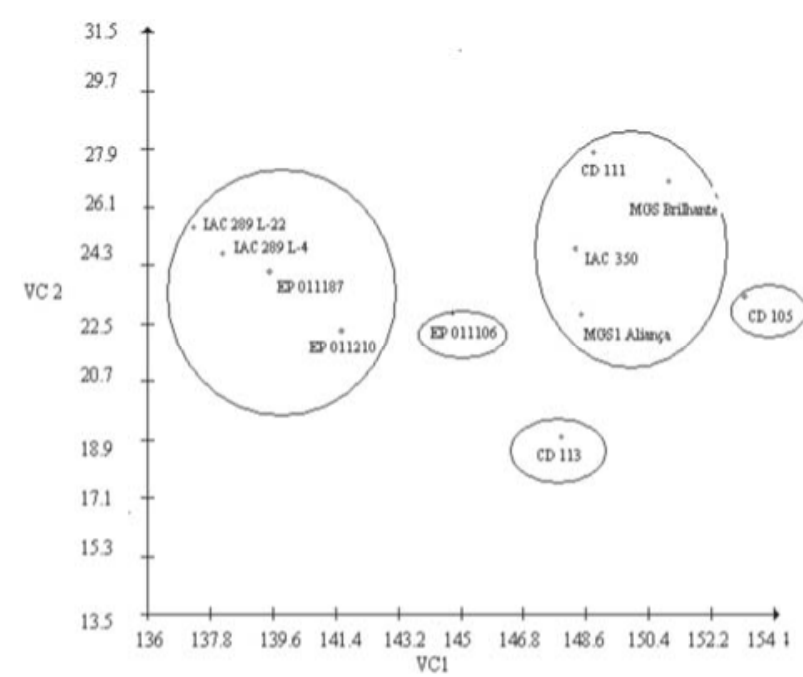

Figura 1. Gráfico de dispersão dos 11 genótipos de trigo avaliados no ano de 2007, em relação às duas primeiras variáveis canônicas.

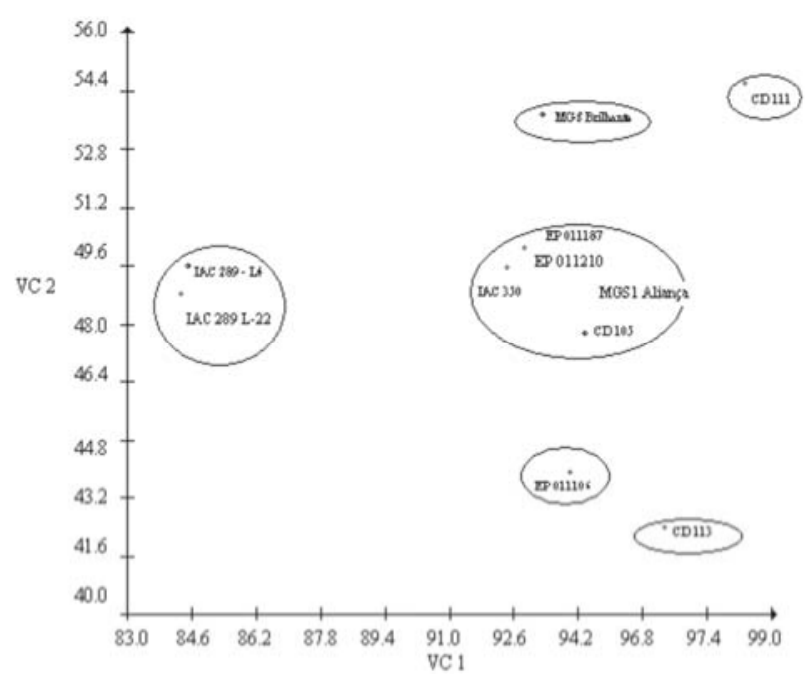

Figura 2. Gráfico de dispersão dos 11 genótipos de trigo avaliados no ano de 2008, em relação às duas primeiras variáveis canônicas.

Rev. Ceres, Viçosa, v. 57, n.6, p. 762-767, nov/dez, 2010 


\author{
011210, IAC 350, MGS 1 Aliança e CD 103; Grupo III - MGS \\ Brilhante; Grupo IV - CD 111; Grupo V - EP 011106; e \\ Grupo VI - CD 113.
}

Esses resultados evidenciaram bastante concordância entre os métodos de análise multivariada utilizados, com pequenas exceções. Bertan et al. (2006), ao avaliarem genótipos de trigo tolerantes ao alumínio, também constataram essa mesma sincronia entre os diferentes métodos para estimar a divergência genética. Isso demonstra a eficiência desses métodos na identificação de genitores promissores para cultivo em sequeiro, sendo de grande importância aos programas de melhoramento de trigo nas regiões do Brasil Central. A existência de alguns resultados contrastantes entre os métodos também foi verificada por Teixeira et al. (2004) ao avaliar a divergência genética entre cultivares de feijão-de-vagem. Essas pequenas discordâncias são completamente possíveis e aceitáveis, uma vez que os métodos se baseiam em princípios distintos para sua estimação.

\section{CONCLUSÕES}

Os genótipos IAC 289-L22 e IAC 289-L4, por serem provenientes de seleção dentro do cultivar IAC 289, foram os mais similares, independentemente do método de avaliação da dissimilaridade empregado, nos dois anos de cultivo .

Os genótipos CD 113 e EP 011106 foram os mais divergentes. São genitores promissores em cruzamentos para obtenção de materiais adaptados e com boas características em cultivo de sequeiro. São interessantes para cruzamentos entre si e com os demais genótipos avaliados.

\section{REFERÊNCIAS}

Bered F, Neto JFB, Rocha BM, Pegoraro DG, Vacaro E \& Carvalho FIF (2002) Caracterização de germoplasma de trigo por meio dos caracteres adaptativos ciclo e estatura. Pesquisa Agropecuária Brasileira, 37:145-150.

Bertan I, Carvalho FIF, Oliveira AC, Silva JAG, Benin G, Vieira EA, Silva GO, Hartwig I, Val6rio IP \& Finatto T (2006) Dissimilaridade genética entre genótipos de trigo avaliados em cultivo hidropônico sob estresse por alumínio. Bragantia, 65:55-63.

Bertan I, Vieira EA, Carvalho FIF, Oliveira AC, Scheeren PL, Olivo F (2007) Variabilidade genética em trigo aferida por meio da distância genealógica e morfológica. Scientia Agraria, 8: 6774.

Brasil. Ministério da Agricultura. Regulamento técnico de identidade e de qualidade do trigo. Brasília, Instrução Normativa SARC/ MA $n^{\circ} 7$, de 15 de agosto de 2001. Diário Oficial da Uniao, 2001

Cavariani C \& Souza MA (1983) Características usadas na descrição de cultivares de trigo. Informe Agropecuário, 97:14-19.

Chiorato AF, Carbonell SAM, Colombo CA, Dias LAS \& Ito MF (2005) Genetic diversity of common bean accessions in the germplasm bank of the Instituto Agronômico - IAC. Crop Breeding and Applied Biotechnology, 5:1-9.
CONAB. Acompanhamento da safra brasileira: grãos: quinto levantamento, fevereiro 2008 / Companhia Nacional de Abastecimento. Disponível em: <http://www.conab.gov.br/conabweb/ download/safra/estudo_safra.pdf $>$. Acessado em: 04 de março de 2008.

Cruz CD (2001) Programa Genes: versão Windows; aplicativo computacional em genética e estatística. Viçosa, Imprensa Universitária. 390p.

CRUZ CD \& CARNEIRO PCS (2003) Modelos biom6tricos aplicados ao melhoramento genético. Viçosa, Imprensa Universitária. $585 \mathrm{p}$

Fronza V, Yamanaka CH, Morita MA, Souza MA, Felício JC \& Gomes R (1999) Avaliação de genótipos de trigo, no cultivo de sequeiro, durante os anos de 1997 e 1998, na região do PADAPMG. In: X Reunião da Comissão Centro Brasileira de Pesquisa de Trigo, Uberaba. Ata e Resumos Expandidos, EPAMIG. p.53-55.

Lobato MTV (2006) Desempenho agronômico de genótipos de trigo em diferentes regiões do Estado de São Paulo, no período 2003-2005. Dissertação de Mestrado. Instituto Agronômico de Campinas, Campinas, 160p.

Rao RC (1952) Advanced statistical methods in biometric research. New York, John Wiley and Son. 389p.

Scott AJ \& Knott M (1974) Cluster analysis method for means in the analysis of variance. Biometrics, 30:507-512.

Teixeira AB, Amaral Júnior AT, Rodrigues R, Pereira TNS \& Bressan-Smith RE (2004) Genetic divergence in snap-bean (Phaseolus vulgaris L.) evaluated by different methodologies. Crop Breeding and Applied Biotechnology, 4:57-62. 